\title{
BMJ Open Prevalence and incidence of anti-SARS- CoV-2 antibodies among healthcare workers in Belgian hospitals before vaccination: a prospective cohort study
}

\author{
Laure Mortgat (D) , ${ }^{1,2}$ Kristien Verdonck (D) , ${ }^{3}$ Veronik Hutse, ${ }^{4}$ Isabelle Thomas, ${ }^{4}$ \\ Cyril Barbezange, ${ }^{4}$ Leo Heyndrickx, ${ }^{5}$ Natalie Fischer, ${ }^{4,6}$ Bea Vuylsteke, ${ }^{3}$ \\ Ines Kabouche, ${ }^{1}$ Kevin K Ariën, ${ }^{5,7}$ Isabelle Desombere, ${ }^{4}$ Els Duysburgh ${ }^{1}$
}

To cite: Mortgat L, Verdonck K, Hutse V, et al. Prevalence and incidence of antiSARS-CoV-2 antibodies among healthcare workers in Belgian hospitals before vaccination: a prospective cohort study. BMJ Open 2021;11:e050824. doi:10.1136/ bmjopen-2021-050824

- Prepublication history and additional online supplemental material for this paper are available online. To view these files, please visit the journal online. To view these files, please visit the journal online (http://dx.doi.org/10.1136/ bmjopen-2021-050824).

Received 01 March 2021 Accepted 25 May 2021
Check for updates

(C) Author(s) (or their employer(s)) 2021. Re-use permitted under CC BY-NC. No commercial re-use. See rights and permissions. Published by BMJ.

For numbered affiliations see end of article.

Correspondence to Dr Laure Mortgat; laure.mortgat@sciensano.be

\section{ABSTRACT}

Objectives To describe prevalence and incidence of antiSARS-CoV-2 antibodies among Belgian hospital healthcare workers (HCW) in April-December 2020.

Design Prospective cohort study. Follow-up was originally planned until September and later extended.

Setting Multicentre study, 17 hospitals.

Participants $50 \mathrm{HCW}$ were randomly selected per hospital. HCW employed beyond the end of the study and whose profession involved contact with patients were eligible. 850 HCW entered the study in April-May 2020, $673 \mathrm{HCW}(79 \%)$ attended the September visit and 308 (36\%) the December visit.

Outcome measures A semiquantitative ELISA was used to detect IgG against SARS-CoV-2 in serum (Euroimmun) at 10 time points. In seropositive samples, neutralising antibodies were measured using a virus neutralisation test. Real-time reverse transcription PCR (RT-qPCR) was performed to detect SARS-CoV-2 on nasopharyngeal swabs. Participant characteristics and the presence of symptoms were collected via an online questionnaire. Results Among all participants, $80 \%$ were women, $60 \%$ nurses and $21 \%$ physicians. Median age was 40 years. The seroprevalence remained relatively stable from April (7.7\% (95\% Cl: $4.8 \%$ to $12.1 \%)$ to September (8.2\% (95\% Cl: $5.7 \%$ to $11.6 \%)$ ) and increased thereafter, reaching $19.7 \%$ (95\% Cl: $12.0 \%$ to 30.6\%) in December 2020.76 of 778 initially seronegative participants seroconverted during the follow-up (incidence: $205 / 1000$ person-years). Among all seropositive individuals, 118/148 (80\%) had a positive neutralisation test, $83 / 147(56 \%)$ presented or reported a positive RT-qPCR, and 130/147 (88\%) reported COVID-19-compatible symptoms at least once. However, only $46 / 73(63 \%)$ of the seroconverters presented COVID-19-compatible symptoms in the month prior to seroconversion.

Conclusions The seroprevalence among hospital HCW was slightly higher than that of the general Belgian population but followed a similar evolution, suggesting that infection prevention and control measures were effective and should be strictly maintained. After two SARS-CoV-2 waves, $80 \%$ of HCW remained seronegative, justifying their prioritisation in the vaccination strategy. Trial registration number NCT04373889
Strengths and limitations of this study

- To our knowledge, this is the first study reporting on SARS-CoV-2 seroprevalence in a nationwide representative sample of hospital healthcare workers (HCW).

- This longitudinal study has a relatively long duration of follow-up (8 months) with until September 2020 (part of the study before its extension) a small number of people lost to follow-up.

- The use of multiple different assays repeated over time reveals a complexity in the profiles of infected participants that would have been missed when looking at one point in time, or using a single test.

- Selection bias might have occurred at recruitment: some hospitals and HCW refused to participate, possibly because of a higher impact of the epidemics locally (work overload, sick leave, etc).

- Loss to follow-up was low but increased over time, especially during the summer months and after the extension of the study beyond September 2020.

\section{INTRODUCTION}

Early December 2019, a novel coronavirus, named SARS-CoV-2, was detected in Wuhan, China, and rapidly spread worldwide. This pandemic, with over 140 million cumulative reported COVID-19 cases and 3 million deaths as of 18 April 2021 led to an unprecedented global health crisis. ${ }^{1}$

Healthcare workers (HCW) represent a highly exposed population, being at the frontline management of patients with COVID-19. Furthermore, if infected, they also pose a risk to the vulnerable patients they care for and to their colleagues. ${ }^{2}$ Their role in the chain of transmission, as well as in ensuring the implementation of appropriate infection prevention and control (IPC) measures is therefore essential. In the vaccination strategies of many countries, HCWs are treated as a priority population. ${ }^{4}$ 
In Belgium, the first imported case of SARS-CoV-2 infection was detected on 3 February, and local transmission was identified early March 2020. ${ }^{5}$ By the end of April 2021, approximately 976000 confirmed cases and 24 000 COVID-19-related deaths had been reported, while Belgian hospitals had admitted a total of 69400 patients with COVID-19. ${ }^{6}$ Several measures have been implemented in hospitals in the hope of limiting transmission. At the peak of the first COVID-19 wave, from mid-March till May 2020, all non-urgent admissions and consultations were suspended. ${ }^{7}$ From mid-April 2020 onwards, universal use of surgical masks was recommended to all HCW likely to be in contact with a COVID-19 case while FFP2 masks were reserved for aerosolising procedures. ${ }^{8}$ The working conditions for HCWs were different during the second wave, from September till December 2020, because regular, non-COVID-19 related care continued and because there were no structural shortages anymore of personal protective equipment. As in other countries, testing strategies and case definition changed over the months, and still today, HCW can only be tested for SARS-CoV-2 if they show well-defined symptoms. ${ }^{9}$ As individuals can carry and transmit the virus without exhibiting any symptoms, ${ }^{10} 11$ a potentially high proportion of cases in this population were therefore never identified.

By April 2020, nearly no data on SARS-CoV-2 infection among HCW in Belgium and in other countries was available, although casualties among medical doctors and nurses were being reported in the media. ${ }^{12}{ }^{13}$ Similarly, data on the proportion of asymptomatic infections among HCW was scarce. Assessing the burden and the clinical presentation of the disease in this high-risk population appeared crucial to reduce secondary virus transmission within this setting. We, thus, started a prospective cohort study end of April 2020, aiming to follow the prevalence and incidence of anti-SARS-CoV-2 antibodies among Belgian hospital HCW throughout the epidemic, in order to guide IPC measures in hospitals and support planning of healthcare resources. In addition, we sought to investigate the presence of symptoms, positive PCR results and neutralising antibodies in seropositive participants, and to describe these variables over time in seroconverters. In this paper, we present the findings up to the end of December 2020, before the start of the vaccination of HCW in Belgium mid-January 2021.

\section{METHODS}

\section{Study design}

This is an observational prospective cohort study describing a random sample of HCW employed in Belgian hospitals. We followed the Strengthening the Reporting of Observational Studies in Epidemiology (STROBE) recommendations to prepare the report. ${ }^{14}$ The completed STROBE checklist is available (see online supplemental file 1).

\section{Study population}

We used two-stage cluster sampling to obtain a random sample of $850 \mathrm{HCW}$. In the first stage, 17 hospitals were selected out of a complete list of 103 general hospitals in Belgium, ${ }^{15}$ using a random procedure with probability proportional to size. The number of hospital beds was taken as a proxy for size, that is, hospitals with more beds had a higher probability to be selected than hospitals with fewer beds. The second stage took place within each of the 17 included hospitals. A local study coordinator randomly selected 50 individuals out of a list of all eligible HCW. To be eligible, HCW had to have an employment contract with the hospital covering at least the length of the study and they had to have contact with patients, be it in COVID-19-dedicated or other wards. If a selected HCW did not want to participate, he/she was replaced by the next person on the list.

Participants were recruited in April and May 2020, at the time of the peak of the first SARS-CoV-2 wave in Belgium. Data were collected every 2 weeks during the first month and then monthly. Follow-up was originally planned until September 2020, but was later extended. The selection procedures and baseline characteristics of the participants are described in more detail elsewhere. ${ }^{16}$

\section{Assessment of anti-SARS-CoV-2 antibodies}

The main outcome was the presence of IgG antibodies against SARS-CoV-2 in serum samples collected at each of the 10 study visits. This was measured using a commercially available ELISA that captures anti-S1 (spike subunit 1) antibodies (Euroimmun anti-SARS-CoV-2 IgG ELISA, reference EI 2606-9601 G, Medizinische Labordiagnostika AG).${ }^{17}$ As recommended by the manufacturer, we used a stringent cut-off to consider a test result as positive (ratio $\geq 1 \cdot 1$; NCT04373889). Studies set up to evaluate the accuracy of this ELISA obtained point estimates for the sensitivity between $88 \%$ and $93 \%$ using samples obtained at least 2 weeks after clinical or molecular diagnosis, and point estimates for the specificity between $96 \%$ and $99 \% .{ }^{18}{ }^{19}$ In our study, ELISA testing was carried out at the laboratories of Sciensano (national public health institute of Belgium).

\section{Assessment of covariates}

Presence of symptoms compatible with COVID-19

At each study visit, the participants were invited to complete an online questionnaire covering the period between the previous and the current visit. We considered that a participant had symptoms compatible with COVID-19 if, during that period, he/she reported to have experienced at least one of the following symptoms: cough, shortness of breath, chest pain, loss of smell or taste; or at least two of the following symptoms: fever, muscle pain, fatigue, running nose, sore throat, headache, acute mental confusion or diarrhoea. ${ }^{9}$

SARS-CoV-2 molecular test results

Nasopharyngeal swabs for molecular testing were taken at each study visit. The samples were transported to the 
Sciensano laboratories or the Institute of Tropical Medicine, Antwerp, where real-time reverse transcription PCR (RT-qPCR) was done, targeting the $\mathrm{E}$ gene and using a $\mathrm{Ct}$ cut-off of $40 .^{20}$ In addition, as part of the online questionnaire, we asked at each study visit if the participants had received a positive RT-qPCR result outside our study; and if that was the case, on what date.

Virus neutralisation test results

For samples with a positive ELISA result, the level of neutralising antibodies was measured using a virus neutralisation test. ${ }^{21-23}$ This test uses Vero cells, which are highly susceptible to infection with coronaviruses and show clear cytopathic effects when they are infected. The result of the neutralisation test is expressed as the serum titre of antibodies needed to neutralise $50 \%$ of the SARS-CoV-2 in vitro infection (NT50). If this titre was 1:50 or higher, the corresponding serum sample was defined as positive on the neutralisation test.

\section{Study size}

The prevalence of anti-SARS-CoV-2 antibodies among HCW was unknown at the time this study was designed. The sample size calculation was based on an estimated seroprevalence of $50 \%$, a desired absolute precision of $5 \%$, and a design effect of 2 . We set the estimated seroprevalence to $50 \%$ because that is a conservative approach (leading to a large sample size) and because we expected to find a seroprevalence of that order of magnitude. ${ }^{24}$ This led to a target sample size of $800 \mathrm{HCW}$, that is, 16 clusters of 50 individuals, but in order to have an additional margin, we decided to include 17 clusters.

\section{Statistical methods}

We first described the prevalence and the incidence rate of positive ELISA results in the study population and inferred these findings to the target population of all HCW in Belgian hospitals. Because of the sampling design, not all HCW had the same probability of being selected. This was corrected at the level of the analysis by using weights consisting of a hospital and an individual HCW component. The computation of the $95 \%$ CIs also took account of the cluster design (detailed description in online supplemental file 1).

Second, we used a time-to-event analysis and a KaplanMeier curve to express the probability of becoming seropositive over time. This analysis only included the participants who were seronegative at baseline and captures the cumulative nature of the seroconversions. Time to event was calculated as the number of days between study inclusion and the collection of the serum sample that gave the first positive ELISA result. Participants who only had negative ELISA results were censored on the date of their last ELISA-negative sample. The timeto-event analysis was corrected for the two-stage cluster sampling design.

Third, we described COVID-19-compatible symptoms, RT-qPCR results and neutralisation results focusing on the study participants who became ELISA positive during the follow-up. We plotted these variables together on timelines to visualise the different patterns of results occurring in this study population.

All statistical analyses were done with $\mathrm{R}$ software (V.3.6.1). To deal with the two-stage sampling design, we used the survey package, ${ }^{25}$ which was developed to analyse data while using weights and accounting for clustering. This package supports the estimation of survival functions by means of a weighted Kaplan-Meier estimator.

\section{Patient and public involvement}

Neither patients (or HCW in this specific study) nor the public were involved in the design of the study, mainly because of the urgency to set it up in the beginning of the pandemic. A local coordinator in each of the selected hospitals contributed to the recruitment, managed the local logistics, and ensured communication between the participants and the researchers throughout the study. Participants who wished to know their individual laboratory test results could obtain these by phone in a pseudonymised manner (using a code). Researchers could then interact with the participants and address their concerns or demands. Seroprevalence results are communicated to the general public via press releases and displayed in a graph that is updated monthly and accessible on the Sciensano dashboard (https://datastudio.google. com/embed/u/0/reporting/7e11980c-3350-4ee3-82913065cc4e90c2/page/ZwmOB).

\section{RESULTS}

\section{General characteristics of hospitals and HCW}

Out of the hospitals that were initially selected, six declined participation because of concurrent SARS-CoV2-testing initiatives at hospital level and concerns about the study-related logistical burden. They were replaced by six other hospitals following the same random selection procedure. The final sample of 17 hospitals included 10 hospitals located in Flanders, five in Wallonia, and two in Brussels. The sample included three university hospitals, two general hospitals with similar characteristics to university hospitals, and 12 general hospitals without university characteristics. The study was initiated in 14 hospitals on 25 April, in two hospitals on 10 May (second time point), and in one hospital on 25 May 2020 (third time point).

The total number of eligible HCW employed in the study hospitals was 24019 , and 850 of them were randomly selected and included in the study. An overview of the baseline characteristics of the study population is given in online supplemental file 2). In summary, their median age was 40 years (interquartile range (IQR) 32-49 years); 677 (80\%) were women; $504(60 \%)$ were nurses; 175 (21\%) were physicians; and 166 (20\%) had another profession. The study participants had been working for a median of 14 years (IQR 7-24 years). More than half of them $(461 / 850 ; 61 \%)$ had a full-time job. 


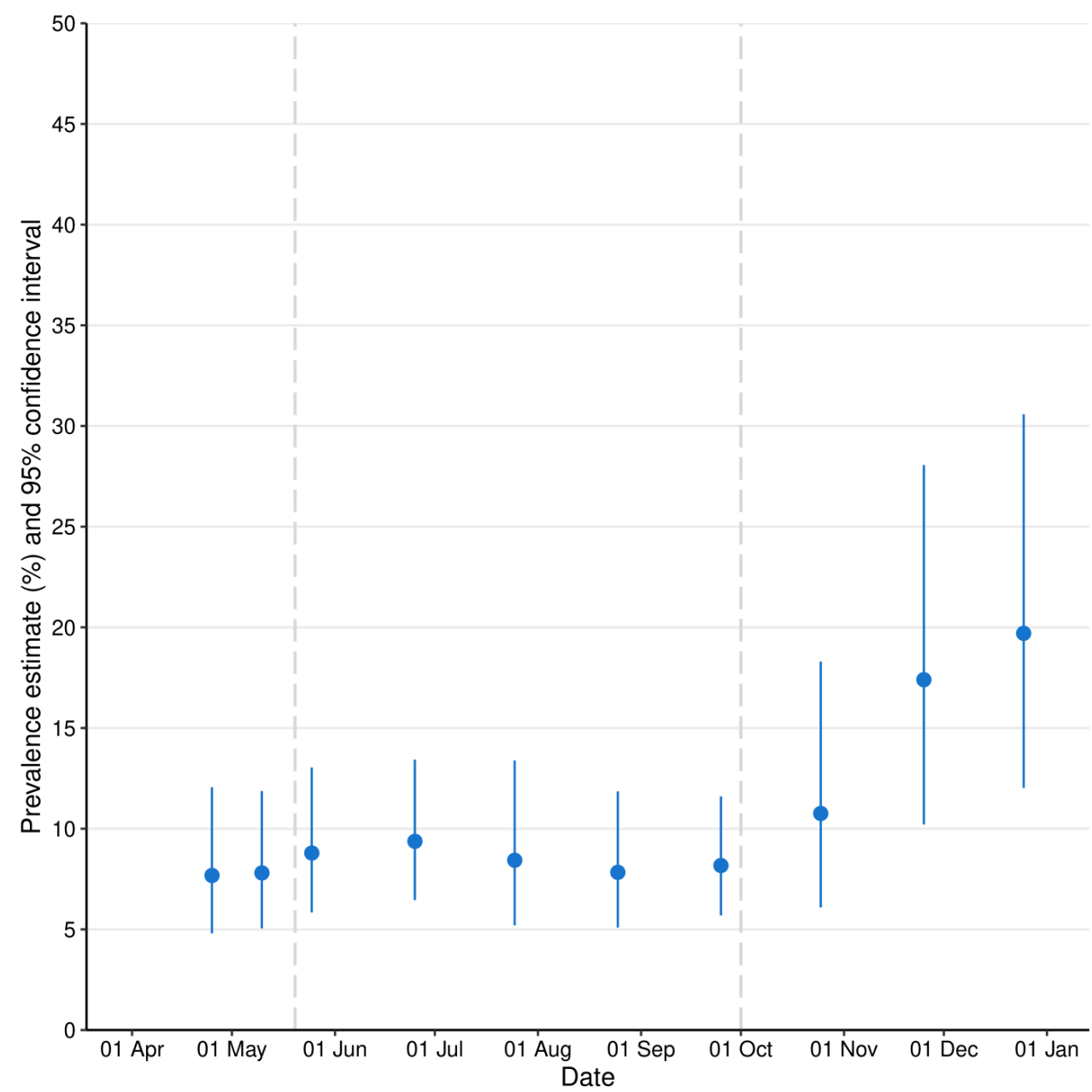

Figure 1 Estimated prevalence of SARS-CoV-2 seropositivity among healthcare workers in Belgian hospitals over a period of 8 months. Individuals with one positive Euroimmun test result are considered to be seropositive for anti-SARS-CoV-2 IgG antibodies. The estimates are based on a cohort of 850 healthcare workers followed up over time and are corrected for the twostage cluster sampling design. In the period between the dashed grey lines, the 17 selected hospitals participated in the study. Before the first dashed line, not all hospitals had started recruitment (two hospitals joined the study on the second time point and one hospital on the third time point). After the second dashed line, five hospitals dropped out of the study.

Until the end of September 2020, half of the participants $(408,48 \%)$ attended all scheduled follow-up visits and 673 HCW $(79 \%)$ were present on the visit of 25 September. From that date onwards, follow-up continued in only 12 of the 17 study hospitals and participation rates decreased (online supplemental file 3): 308 participants $(36 \%)$ were present on the visit of end December.

\section{Prevalence and incidence rate of anti-SARS-CoV-2 antibodies}

During the first 5 months of the study, the seroprevalence remained relatively stable. After adjustment for the sampling design, the estimated seroprevalence among HCW in Belgian hospitals was $7.7 \%$ (95\% CI: $4.8 \%$ to $12.1 \%$ ) in April 2020 and $8.2 \%$ (95\% CI: $5.7 \%$ to $11.6 \%$ ) in September 2020. From that point onwards, the seroprevalence increased and reached $19.7 \%$ (95\% CI: $12.0 \%$ to $30.6 \%$ ) by the end of December 2020 (figure 1).

To estimate the incidence of developing antiSARS-CoV-2 antibodies (seroconversion), we focused on participants who were seronegative at baseline and analysed participant time instead of calendar time. 778
$(91.5 \%)$ of the 850 participants were seronegative at baseline (which could be on 25 April, 10 May or 25 May 2020, depending on the hospital). The median duration of serological follow-up in this group was 153 days (IQR 123-244 days). 76 of the 778 initially seronegative participants seroconverted during a total follow-up duration of 371 person-years at risk. This corresponds to an incidence rate of 205 per 1000 person-years. The probability of becoming seropositive over time is shown in the KaplanMeier curve (figure 2). The median age of the seroconverters was 40 years (IQR 33-51); 59 were women; 15 were physicians; 43 were nurses and 17 had another profession (profession was missing for one participant).

\section{COVID-19-compatible symptoms, RT-qPCR results and neutralisation test results}

$68 \%$ of the participants $(574 / 849)$ reported to have had COVID-19-compatible symptoms: $20 \%$ (171/849) had these symptoms only in the period between the start of the pandemic in Belgium and the start of our study; for $27 \%(227 / 849)$, the symptoms started prior to the study 




Numbers of healthcare workers at risk

Figure 2 Kaplan-Meier curve showing the probability of becoming seropositive over time. The Kaplan-Meier analysis includes 778 healthcare workers who were seronegative at baseline. The solid line indicates the probability of becoming seropositive over time. The shaded area represents the $95 \%$ confidence bands.

and continued during the follow-up; and 21\% (176/849) developed their first COVID-19-compatible symptoms during the follow-up.

24 HCW $(3 \%)$ tested positive on an RT-qPCR test conducted as part of the study: eight in April, four in May, two in September, seven in October and three in November 2020. 13 out of these 24 HCW reported that they had tested RT-qPCR positive outside the study as well. Seventy-five additional HCW mentioned a positive RT-qPCR result elsewhere, but remained RT-qPCR negative in our study. 34 of these external molecular diagnoses occurred prior to the start of our study. One participant reported two positive RT-qPCR results outside the study, one in May and another in October 2020. Apart from this person, no other HCW experienced more than one documented episode of SARS-CoV-2 infection.

Table 1 gives an overview of the different test results among HCW with and without COVID-19-compatible symptoms. Overall, $150 \mathrm{HCW}$ were diagnosed with active or past SARS-CoV-2 infection through our study: $126 \mathrm{had}$ a positive ELISA only (of whom 61 reported a positive RT-qPCR elsewhere), 2 had a positive RT-qPCR only and
22 tested positive both on ELISA and RT-qPCR. Fourteen additional HCW reported a positive RT-qPCR elsewhere but did not have molecular or serological evidence of infection in our study.

Whole virus neutralisation test results were positive (at any point in time) in 63 out of the $72 \mathrm{HCW}(88 \%)$ who were ELISA-seropositive at baseline and in 55 out of the 76 HCW (72\%) who seroconverted during the study. Figure 3 visualises the presence of COVID-19-compatible symptoms and all available laboratory test results on a timeline for three participants who represent three types of result patterns. Participant A had a confirmed molecular diagnosis of COVID-19 and a clear and consistent serological response. Participant B had positive results on the ELISA and neutralisation tests but no molecular proof of SARS-CoV-2 infection during the study. Participant $\mathrm{C}$ had an unexpected pattern: the ELISA and neutralisation test results did not agree; the ELISA response disappeared after a few months; and the seroconversion occurred before the positive molecular test result.

Among the 76 participants who seroconverted, there were 44 individuals (58\%) with consistent molecular and 
Table 1 Overview of serological and molecular test results among healthcare workers with and without COVID-19-compatible symptoms

\begin{tabular}{|c|c|c|c|c|}
\hline & $\begin{array}{l}\text { ELISA positive at } \\
\text { baseline }\end{array}$ & $\begin{array}{l}\text { Seroconversion } \\
\text { during follow-up }\end{array}$ & $\begin{array}{l}\text { ELISA negative at } \\
\text { all time points }\end{array}$ & Total \\
\hline & n (column \%) & n (column \%) & n (column \%) & n (column \%) \\
\hline RT-qPCR positive in the study $†$ & $5(7) \ddagger$ & $16(21) \S$ & $2(0) \rrbracket$ & $23(3)$ \\
\hline RT-qPCR positive elsewhere only* & $26(36)$ & $32(43)$ & $13(2)$ & $71(8)$ \\
\hline RT-qPCR positive in the study & $0(0)$ & $1(1)$ & $0(0)$ & $1(0)$ \\
\hline RT-qPCR positive elsewhere only* & $1(1)$ & $2(3)$ & $1(0)$ & $4(0)$ \\
\hline RT-qPCR negative & $7(10)$ & $6(8)$ & $257(37)$ & $270(32)$ \\
\hline Total & 72 & $75 f$ & 702 & $849^{* *}$ \\
\hline
\end{tabular}

${ }^{*}$ At any point between the start of the pandemic and the last study visit as reported by the participants in the questionnaire.

†At any of the 10 scheduled study visits.

$\ddagger$ Three out of five HCW tested RT-qPCR positive both in the study and elsewhere.

§Nine out of $16 \mathrm{HCW}$ tested RT-qPCR positive both in the study and elsewhere.

ПOne out of two HCW tested RT-qPCR positive both in the study and elsewhere.

${ }^{\star *}$ One HCW with seroconversion during follow-up is missing because he/she did not fill out the questionnaire.

HCW, healthcare worker; RT-qPCR, real-time reverse transcription PCR.

serological proof of SARS-CoV-2 infection (pattern similar to that of participant A). Seventeen out of $76(22 \%)$ had positive serological results only (similar to participant B). The remaining 15 participants (20\%) had various unexpected patterns (similar to participant $\mathrm{C}$ ), that is, discordant results, quickly waning antibody responses, and/or a puzzling order of events (detailed timelines in online supplemental file 4 ).

The relation between symptoms and seroconversion could be assessed in 73 participants who completed the questionnaire: 46 out of 73 HCW (63\%) had COVID-19compatible symptoms starting within 4 weeks before they seroconverted; 18 (25\%) reported COVID-19-compatible symptoms but the timing did not coincide with the seroconversion (symptoms after or more than 4 weeks before seroconversion) and 9 (12\%) did not report symptoms at all.

\section{DISCUSSION}

In this longitudinal study, we followed up SARS-CoV-2 IgG seroprevalence in a national representative cohort of 850 hospital HCW. Our findings indicate that this prevalence fluctuated around 8\% between April and September 2020 and then increased up to $20 \%$ in December. Out of the 148 participants who showed evidence of a serological response, $56 \%$ were also positive by PCR (inside or outside the study), $80 \%$ had neutralising antibodies and $88 \%$ presented COVID-19 compatible symptoms at any point since the beginning of the epidemic. During the 8 months of follow-up, we documented seroconversion in 76 participants (incidence rate of 205 per 1000 personyears), of whom $67 \%$ were also positive by PCR (in- or outside the study), $72 \%$ had neutralising antibodies and 63\% reported recent COVID-19-compatible symptoms.

The course of the COVID-19 outbreak in Belgium in 2020 was characterised by two clearly distinct waves, with peaks in April and October. Our study started just after the peak of the first wave and ended 2 months after the peak of the second wave: it captures periods of low (May to August) and high transmission (September to November) ${ }^{26}$ This is reflected in our data: the seroconversions we observed occurred essentially in the first and the last months of follow-up.

To our knowledge, this is the first study reporting on SARS-CoV-2 seroprevalence in a nationwide representative sample of hospital HCW, allowing us to infer our findings to this population. Until September 2020 (the part of the study before its extension), the number of participants lost to follow-up was low. Furthermore, the total duration of follow-up was long (until end December 2020), which provides a clear view of the serological status of HCW just before the start of the vaccination campaign (in January 2021).

Another strength of this study is the combination of multiple different assays repeated over time. The different patterns observed reveal a complexity that would have been missed when looking at one point in time or using a single test. This highlights the importance of interpreting test results cautiously, and needs to be taken into account in the development of individual and public health diagnostic and screening strategies. The incomplete overlap of results of the ELISA and neutralisation test could be explained by the fact that virus neutralisation testing measures a subfraction of anti-SARS-CoV-2 antibodies 

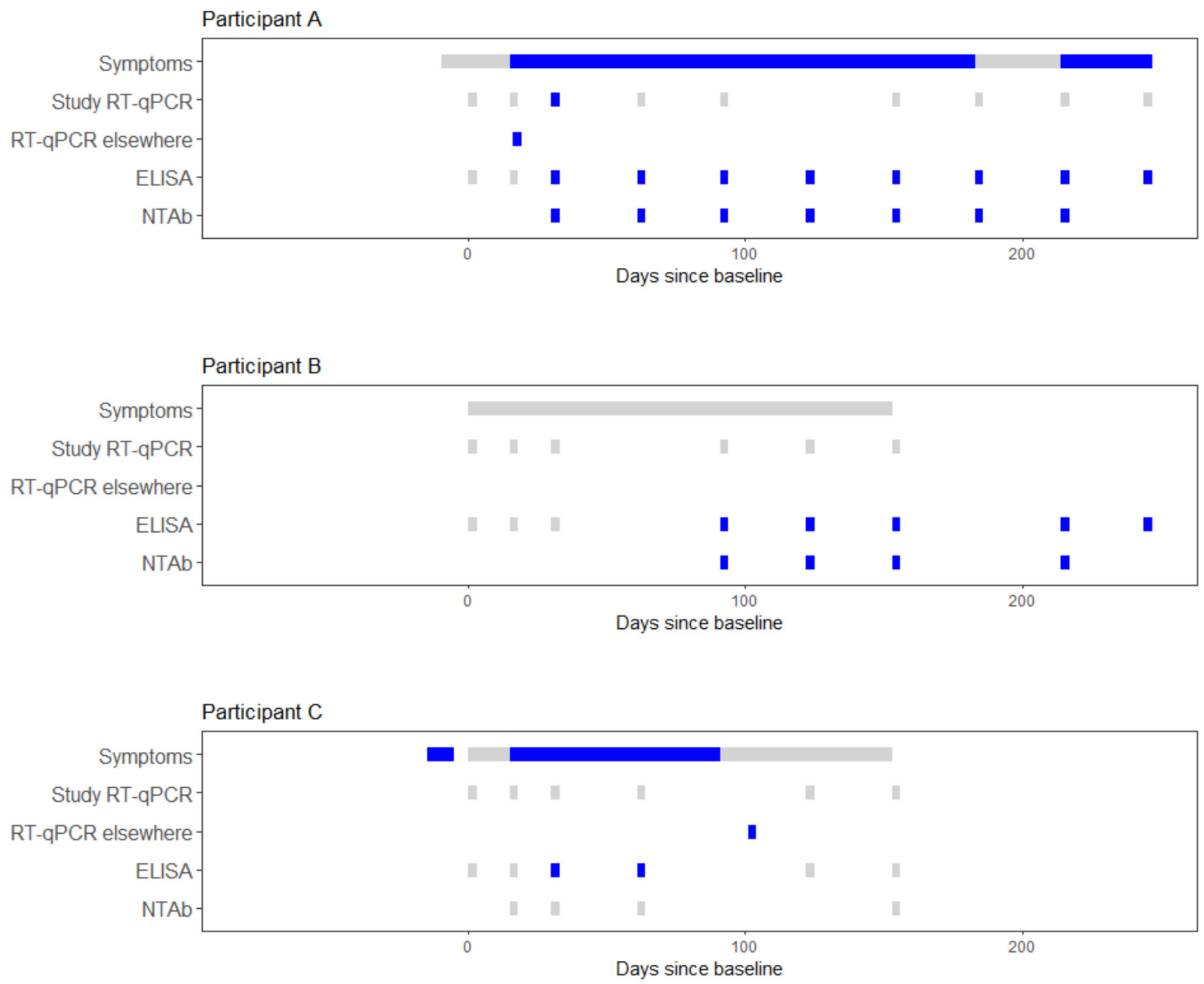

Figure 3 Timeline indicating symptoms, RT-qPCR, ELISA and neutralisation test results of three selected participants who seroconverted during follow-up. Grey colour indicates absence of symptoms or negative test results. Blue colour indicates presence of symptoms (prior to a study visit) and positive test results (on the day of a study visit). Participant A had a confirmed molecular diagnosis of SARS-CoV-2 infection and consistent serological response. Participant B presented a consistent serological response but had no molecular proof of SARS-CoV-2 infection. Participant $\mathrm{C}$ had an unexpected pattern of results (discordant ELISA and neutralisation test results, waning antibody response, and puzzling timing of events). The timelines of 15 participants with unexpected patterns are available in online supplemental file 4. NTAb, virus neutralisation test; RT-qPCR, realtime reverse transcription $\mathrm{PCR}$.

with neutralising quality whereas ELISA measures both non-neutralising and neutralising antibodies. Another explanation could be that some individuals react falsepositive in SARS-CoV-2 ELISA because of S1-specific cross-reactivity induced by antibodies against common cold coronaviruses.

Our study also has several limitations. Selection bias might have occurred at recruitment, as some hospitals and HCW refused to participate. We cannot exclude that some hospital or participant refusals might be due to a work overload because of a higher impact of the epidemic locally. Equally, some HCW might have been on sick leave during recruitment, possibly due to a COVID-19 infection, which could underestimate our results. Although the drop-out rate was low in the first months of follow-up, some participants missed a study visit during the summer holiday period. More importantly, when the study was extended beyond September 2020, five hospitals as well as 177 HCW from the 12 remaining hospitals discontinued their participation. We have no direct indications that the decision to participate in the extension was linked to COVID-19 risk. Nevertheless, we acknowledge that the reduction of the study population may have affected its representativeness. There are also limitations associated with survival analysis and interval censoring: the approximation of seroconversion dates can lead to an underestimation of the hazard of seroconversion. 
Most seroprevalence studies among HCW conducted so far are prevalence or cross-sectional studies, as shown by two recent meta-analyses ${ }^{2728}$ which identified only two ${ }^{29} 30$ and three cohort studies ${ }^{30-32}$ out of respectively 28 and 49 studies reporting data on seroprevalence. Furthermore, these cohort studies presented data corresponding to a follow-up of 1 month or less. All of the included studies used a sampling strategy that did not allow national representativeness; they were mainly single centre studies using a convenience sample. Although findings of these two systematic reviews were highly heterogeneous across studies, countries or regions, our results up to September 2020 are consistent with the pooled seroprevalence found of $7 \%$ (95\% CI: 7 to 15 ) and $8.7 \%$ (95\% CI: $6.7 \%$ to $10.9 \%) .{ }^{27}{ }^{28}$ However, we found a substantially higher seroprevalence in the last 3 months of the study (up to $20 \%$ ). Other Belgian studies carried out among HCW in single hospitals in the period of April to June have observed seroprevalences of $6.4 \%,{ }^{33} 12.0 \%{ }^{30}$ and $14.6 \%,{ }^{34}$ which are compatible with our results.

The evolution of the seroprevalence among the HCW in this study was very similar to that in the general Belgian population. Among blood donors and in residual blood samples (taken as a proxy for the general population), the seroprevalence was around $5 \%$ after the first wave (compared with $8 \%$ in our study), remained stable until September, and then increased up to $16 \%$ by the end of the second wave (compared with $20 \%$ in our study). ${ }^{35} 36$ A study of primary healthcare providers in the region of Flanders revealed the same trend: here, the seroprevalence remained stable between June and September (around 5\%) and increased substantially thereafter (up to $13 \%$ in December). ${ }^{37}$ The consistently higher estimates in our study compared with the general population confirm the occupational risk for SARS-CoV-2 infection among HCW. Nevertheless, the difference between HCW and the general population (about 4\%) was smaller than we had expected and did not increase over time, which suggests that HCW in Belgian hospitals managed to implement relatively adequate personal protection measures.

Seroprevalence studies are important in assessing the proportion of people affected by the pandemic, in the general population but also in highly exposed groups. So far, and with a few exceptions (eg, high-risk contact), only symptomatic HCW can be tested by PCR in Belgium, while serological tests are restricted to high-risk personnel according to local risk management. ${ }^{9}$ However, we found that $37 \%$ of the participants who seroconverted during follow-up did not present recent symptoms (in the month prior to seroconversion) compatible with COVID-19, and were thus missed by the current testing strategy. In addition to the systematic use of IPC measures, early identification and isolation of infected individuals remains crucial to stop the pandemic, even though the role of asymptomatic transmission is still unclear. ${ }^{27}$

Although the understanding of the immune responses to SARS-CoV-2 is growing rapidly, the actual correlate of protection has not been defined yet. More thorough investigation is therefore needed, especially in the light of vaccination. There is conflicting evidence about waning of antibodies, but our study points out that antibodies persist for at least 4 months in more than $90 \%$ of seropositive individuals. ${ }^{38}$ This is consistent with a recent estimation of the duration of Spike IgG antibodies, which seem to decline only modestly after $6-8$ months. ${ }^{39}$ Additionally, evidence is lacking on the protective role of antibodies against reinfection, as well as the role of T-cell mediated immunity. Our study will be extended until April 2021, offering a unique opportunity to follow reinfections and the duration of immune responses after natural infection and vaccination.

In conclusion, the seroprevalence among hospital HCW was slightly higher than that of the general population in Belgium, but followed a similar evolution over time. This suggests that the IPC measures in the hospitals were relatively effective and should be strictly maintained. After two SARS-CoV-2 waves in Belgium, $80 \%$ of the hospital workers were still seronegative, justifying their prioritisation in the vaccination campaign which started in January 2021.

\section{Author affiliations}

${ }^{1}$ Department of Epidemiology and Public Health, Sciensano, Brussels, Belgium ${ }^{2}$ European Programme for Intervention Epidemiology Training (EPIET), ECDC, Solna, Sweden

${ }^{3}$ Department of Public Health, Institute of Tropical Medicine, Antwerp, Belgium ${ }^{4}$ Department of Infectious Diseases in Humans, Sciensano, Brussels, Belgium ${ }^{5}$ Department of Biomedical Sciences, Institute of Tropical Medicine, Antwerp, Belgium

${ }^{6}$ European Public Health Microbiology Training (EUPHEM), ECDC, Solna, Sweden ${ }^{7}$ Department of Biomedical Sciences, University of Antwerp, Antwerp, Belgium

Correction notice This article has been corrected since it was published. Affiliation for Kevin K Ariën has been corrected.

Acknowledgements We want to thank each of the participating hospitals and their local coordination team for their logistical support: AZ Damiaan ziekenhuis, Oostende-AZ Delta- AZ Jan Yperman Ziekenhuis: Apr. Klinisch bioloog Stijn Jonckheere, study nurse Melissa Devos and hospital hygiene team led by Dirk Vanrenterghem. AZ Sint-Lucas Brugge: Dr. Johan Robbrecht, Head of Department of Clinical Biology; Lislot Mommerency, Coordinator of Clinical Studies; Larissa Staelens, Study Coordinator. AZ West: Leen Pollet, MD; Klaas Vandevyvere, MD; Lieve Debruyne, hospital hygiene nurse - CHIREC-hôpital de Waterloo-BraineI'Alleud-Europe Hospitals, Brussels : Olivier Lang (nurse), Matthieu Pierre Rutgers, MD-Grand Hôpital de Charleroi (GHdC), toutes équipes volontaires-Clinique Saint Pierre 0ttignies: Dr Grégoire Wieërs, Dr Valérie Selosse, Catherine Georges, Isabelle Vanbellinghen, Martine Gérard. Ghent University Hospital, Dept Internal Medicine: Steven Callens, MD/PhD ; Sophie Vanherrewege (study nurse). Jessa Ziekenhuis : Apr Biol Sara Vijgen and Dr Koen Magerman, in collaboration with MENSURA: Dr Nele Van Loon and Mathieu Verbrugghe. Mariaziekenhuis Noord-Limburg-Professor Dr Erika Vlieghe (Antwerp University Hospital / Institute of Tropical Medicine)-Dr Patrick. Soentjens (Institute of Tropical Medicine / Antwerp University Hospital). Vivalia-Centre Hospitalier de l'Ardenne: Gerald Malempré ; Sylvie Thomas ; Marie André, MD; Pierre Yves Machurot, MD. ZNA Middelheim-Dr Benjamin Scott and study team-CHR Verviers East Belgium. For her help in providing results feedback to participants, we also thank Veerle Boonen, MS, Department of Epidemiology and Public Health, Sciensano, Brussels, Belgium. For their technical assistance: Department of Infectious diseases in humans, Sciensano, Brussels, Belgium: Caroline Rodeghiero, Fabienne Jurion, Assia Hamouda, Ilham Fdillate, Reinout Van Eycken, Mona Abady, Aurélie Francart, Jeannine Weyckmans, Marjorie Jacques, Vera Verburgh, Sophie Lamoral. Biomedical dept. ITM: Anne Hauner, Betty Willems, Sandra Coppens, Johan Michiels, Koen Bartholomeeusen, Ann Ceulemans. Central lab for Clinical biology: Isabelle Micalessi, Kadrie Ramadan, Lara Balcaen, Karin Van Looveren, Jolien Baeyens, Vicky Cuylaerts, Hilde Smet, Saïd Abdellati, Amina Taïbi. 
For their logistical assistance in preparing the kits: Kim Borighem, Vincent Marteau, Pascale Elsocht; and in sample management: Bart De Logi, Nathalie Stassen. Thanks to Sooria Balasegaram, MD; for her support, review and input at all stages of the study. Our gratitude finally goes to all the study participants.

Contributors LM and ED are guarantors of the study. They had full access to the data, accept responsibility for the conduct of the study, and decision to submit for publication. Concept, design, protocol writing: LM, ED, KKA and ID. Logistical coordination: ED, LM, VH, IK and LH. Administrative, technical or material support: all authors.Biological samples collection, transport and analysis: KKA, CB, ID, NF, $\mathrm{LH}, \mathrm{VH}$ and IT. Epidemiological data collection, cleaning and analysis: LM, KV and ED. Drafting of manuscript: LM and KV. Manuscript revision: all authors. Funding: ED and ID. Statistical analysis: KV. Supervision: ED. KKA, ID and ED: shared last authorship.

Funding This study was funded by Sciensano, the Belgian institute of public health, Brussels, Belgium. Sciensano was involved in all stages of the study, from conception and implementation to analysis and reporting. Researchers were independent from funders. All authors had full access the data in the study and can take responsibility for the integrity of the data and the accuracy of the data analysis.

Competing interests None declared.

Patient consent for publication Not required.

Ethics approval The study was approved by the Medical Ethics Committee of the University Hospital Ghent (reference: B6702020000036). Written informed consent was obtained from all HCW before enrolment in the study. To guarantee confidentiality, study laboratory results and questionnaires were pseudonymised using unique study codes

Provenance and peer review Not commissioned; externally peer reviewed.

Data availability statement Data are available on reasonable request. The relevant anonymised patient level data as well as statistical code that support the findings of this study are available from the corresponding author on reasonable request.

Supplemental material This content has been supplied by the author(s). It has not been vetted by BMJ Publishing Group Limited (BMJ) and may not have been peer-reviewed. Any opinions or recommendations discussed are solely those of the author(s) and are not endorsed by BMJ. BMJ disclaims all liability and responsibility arising from any reliance placed on the content. Where the content includes any translated material, BMJ does not warrant the accuracy and reliability of the translations (including but not limited to local regulations, clinical guidelines, terminology, drug names and drug dosages), and is not responsible for any error and/or omissions arising from translation and adaptation or otherwise.

Open access This is an open access article distributed in accordance with the Creative Commons Attribution Non Commercial (CC BY-NC 4.0) license, which permits others to distribute, remix, adapt, build upon this work non-commercially, and license their derivative works on different terms, provided the original work is properly cited, appropriate credit is given, any changes made indicated, and the use is non-commercial. See: http://creativecommons.org/licenses/by-nc/4.0/.

\section{ORCID iDs}

Laure Mortgat http://orcid.org/0000-0002-8298-8547

Kristien Verdonck http://orcid.org/0000-0003-1153-4078

\section{REFERENCES}

1 World Health Organisation. COVID-19 Weekly epidemiological update, 2020. Available: https://www.who.int/publications/m/ item/weekly-epidemiological-update-on-covid-19-20-april-2021 [Accessed May 2021].

2 Karlsson U, Fraenkel CJ. Covid-19: risks to healthcare workers and their families. BMJ 2020;m3944.

3 Nguyen LH, Drew DA, Graham MS, et al. Risk of COVID-19 among front-line health-care workers and the general community: a prospective cohort study. Lancet Public Health 2020;5:e475-83.

4 World Health Organisation. Who SAGE roadmap for prioritizing the use of COVID-19 vaccines in the context of limited supply: an approach to inform planning and subsequent recommendations based upon epidemiologic setting and vaccine supply scenarios, November 2020. Available: https://cdn.who.int/media/docs/defaultsource/immunization/sage/covid/sage-prioritization-roadmapcovid19-vaccines_31a59ccd-1fbf-4a36-a12f-73344134e49d.pdf? sfvrsn=bf227443_36\&download=true [Accessed 2021 May].
5 Sciensano. Fact sheet - COVID-19 disease (SARS-CoV-2 virus), April 2021. Available: https://covid-19.sciensano.be/sites/default/files/ Covid19/COVID-19_fact_sheet_ENG.pdf [Accessed May 2021].

6 Sciensano. COVID-19 - epidemiologisch bulletin van 27 April 2021, April 2021. Available: https://covid-19.sciensano.be/sites/default/ files/Covid19/Meest\%20recente\%20update.pdf [Accessed May 2021].

7 de Meeûs $D$, Dardenne L. Le plan urgence déclenché dans tous les hôpitaux belges. La Libre Belgique, March 13, 2020. Available: https://www.lalibre.be/belgique/societe/le-plan-urgence-declenchedans-tous-les-hopitaux-belges-5e6be556f20d5a29c6610885 [Accessed May 2021].

8 Sciensano. Consensus sur l'usage rationnel et correct des masques buccaux en période de pandémie COVID 19, April 2020. Available: https://covid-19.sciensano.be/sites/default/files/Covid19/ consensus $\% 20$ on\%20the\%20use \%20of\%20masks_RMG_FR.pdf [Accessed May 2021].

9 Sciensano. Définition de cas, indications de demande d'un test et déclaration obligatoire de cas COVID-19, December 31, 2020 Available: https://covid-19.sciensano.be/sites/default/files/Covid19/ COVID-19_Case\%20definition_Testing_FR.pdf [Accessed May 2021].

10 Nikolai LA, Meyer CG, Kremsner PG, et al. Asymptomatic SARS coronavirus 2 infection: invisible yet invincible. Int $J$ Infect Dis 2020:100:112-6.

11 He D, Zhao S, Lin Q, et al. The relative transmissibility of asymptomatic COVID-19 infections among close contacts. Int $J$ Infect Dis 2020;94:145-7.

12 teleSUR. Al menos 14 médicos fallecen POR Covid-19 en lquitos, Perú, 2020. Available: https://www.telesurtv.net/news/medicosinfectados-coronavirus-iquitos-peru-20200512-0052.html [Accessed May 2021].

$13 \mathrm{Kim}$ S. Over 100 doctors and nurses have died combating coronavirus across the world. Newsweek, 2020. Available: https:// www.newsweek.com/coronavirus-deaths-infections-doctors-nurseshealthcare-workers-medical-staff-1496056 [Accessed May 2021].

14 von Elm E, Altman DG, Egger M, et al. The strengthening the reporting of observational studies in epidemiology (STROBE) statement: guidelines for reporting observational studies*. Bull World Health Organ 2007;85:867-72.

15 Federal Public Service Health,. Food Chain Safety and, Environment. General Hospitals - Edition 2019. Brussels, 2019. Available: https:// www.health.belgium.be/sites/default/files/uploads/fields/fpshealth_ theme_file/key_data_in_healthcare_gh_v04.pdf [Accessed May 2021].

16 Mortgat L, Barbezange C, Fischer N. SARS-CoV-2 prevalence and seroprevalence among healthcare workers in Belgian hospitals: baseline results of a prospective cohort study. MedRxiv 2020.

17 EUROIMMUN, a PerkinElmer company. Anti-SARS-CoV-2 ELISA IgG Available: https://www.coronavirus-diagnostics.com/documents/ Indications/Infections/Coronavirus/EI_2606_D_UK_A.pdf [Accessed 21 Jun 2020]

18 Deeks JJ, Dinnes J, Takwoingi Y, et al. Antibody tests for identification of current and past infection with SARS-CoV-2. Cochrane Database Syst Rev 2020;6:CD013652.

19 Patel EU, Bloch EM, Clarke W, et al. Comparative performance of five commercially available serologic assays to detect antibodies to SARS-CoV-2 and identify individuals with high neutralizing titers. $J$ Clin Microbiol 2021;59:e02257-20.

20 Corman VM, Landt O, Kaiser M, et al. Detection of 2019 novel coronavirus (2019-nCoV) by real-time RT-PCR. Euro Surveill 2020;25:2000045.

21 World Health Organisation. Who guidelines for the global surveillance of severe acute respiratory syndrome (SARS). updated recommendations October 2004, October 2004. Available: https:// www.who.int/csr/resources/publications/WHO_CDS_CSR_ARO_ 2004_1.pdf?ua=1 [Accessed May 2021].

$22 \mathrm{Ng} \mathrm{DL}$, Goldgof GM, Shy BR, et al. SARS-CoV-2 seroprevalence and neutralizing activity in donor and patient blood. Nat Commun 2020;11:4698.

23 Mariën J, Ceulemans A, Michiels J, et al. Evaluating SARS-CoV-2 spike and nucleocapsid proteins as targets for antibody detection in severe and mild COVID-19 cases using a Luminex bead-based assay. J Virol Methods 2021;288:114025.

24 Sullivan KM. Documentation for sample size for a proportion. OpenEpi: open source epidemiologic statistics for public health, August 29, 2003. Available: https://www.openepi.com/PDFDocs/ SSProporDoc.pdf [Accessed 2021 May].

25 Lumley T. Package 'survey': analysis of complex survey samples, April 03, 2020. Available: https://cran.r-project.org/web/packages/ survey/survey.pdf [Accessed May 2021]. 
26 Sciensano. Belgium COVID-19 epidemiological situation summary. Available: https://epistat.wiv-isp.be/covid/covid-19.html [Accessed May 2021].

27 Gómez-Ochoa SA, Franco OH, Rojas LZ, et al. COVID-19 in healthcare workers: a living systematic review and meta-analysis of prevalence, risk factors, clinical characteristics, and outcomes. Am J Epidemiol 2021;190:161-75.

28 Galanis P, Vraka I, Fragkou D, et al. Seroprevalence of SARS-CoV-2 antibodies and associated factors in healthcare workers: a systematic review and meta-analysis. J Hosp Infect 2021;108:120-34.

29 Houlihan C, Vora N, Byrne T. SARS-CoV-2 virus and antibodies in front-line health care workers in an acute hospital in London: preliminary results from a longitudinal study. MedRxiv 2020.

30 Martin C, Montesinos I, Dauby N, et al. Dynamics of SARS-CoV-2 RT-PCR positivity and seroprevalence among high-risk healthcare workers and hospital staff. J Hosp Infect 2020;106:102-6.

31 Houlihan CF, Vora N, Byrne T, et al. Pandemic peak SARS-CoV-2 infection and seroconversion rates in London frontline health-care workers. Lancet 2020;396:e6-7.

32 Pallett SJC, Rayment M, Patel A, et al. Point-Of-Care serological assays for delayed SARS-CoV-2 case identification among healthcare workers in the UK: a prospective multicentre cohort study. Lancet Respir Med 2020;8:885-94.
33 Steensels D, Oris E, Coninx L, et al. Hospital-Wide SARS-CoV-2 antibody screening in 3056 staff in a tertiary center in Belgium. JAMA 2020;324:195-7.

34 Blairon L, Mokrane S, Wilmet A, et al. Large-Scale, molecular and serological SARS-CoV-2 screening of healthcare workers in a 4-site public hospital in Belgium after COVID-19 outbreak. J Infect 2021;82:159-98.

35 Sciensano. Belgium COVID-19 studies: seroprevalence in blood donors. Available: https://epistat.wiv-isp.be/covid/covid-19.html [Accessed May 2021].

36 Herzog S, De Bie J, Abrams S. Seroprevalence of IgG antibodies against SARS coronavirus 2 in Belgium: a prospective crosssectional study of residual samples. MedRxiv 2021.

37 Mariën J, Ceulemans A, Bakokimi D. Prospective SARS-CoV-2 cohort study among general practitioners during the second COVID-19 wave in Flanders, Belgium. MedRxiv 2021.

38 Duysburgh E, Mortgat L, Barbezange C, et al. Persistence of IgG response to SARS-CoV-2. Lancet Infect Dis 2021;21:163-4.

39 Dan JM, Mateus J, Kato Y, et al. Immunological memory to SARS-CoV-2 assessed for up to 8 months after infection. Science 2021;371:eabf4063. 